\title{
Estudo de diferentes taxas de deformação do comportamento mecânico a quente do aço inoxidável duplex UNS S32304
}

\author{
Caetano, G. A. ${ }^{1 *}$; Orlando, M. T. D. ${ }^{1}$; Rossi, J.L. ${ }^{2}$ \\ 1 Programa de Pós-Graduação em Engenharia Mecânica, Universidade Federal do Espírito Santo, Vitória, ES, Brasil. \\ 2 Instituto de Pesquisas Energéticas e Nucleares, IPEN, São Paulo, SP, Brasil. \\ *e-mail: biaksascki@ufes.br
}

\begin{abstract}
Resumo
O aço inoxidável duplex UNS S32304 tem sido considerado candidato para construção de um receptáculo para transporte de material radioativo, para atender futuras necessidades do Reator Multipropósito Brasileiro (RMB) em construção. Os receptáculos devem apresentar resistência mecânica elevada para evitar falha e, consequentemente, exposição do material radioativo, considerando como a condição mais crítica um acidente aéreo com explosão. Neste trabalhado, avaliou-se o efeito de diferentes taxas de deformação no comportamento tensão-deformação de chapas de UNS 532304 com 1,8 mm de espessura, simulando um acidente aéreo. Os corpos de prova foram submetidos a ensaios de tração a quente no simulador termomecânico Gleeble ${ }^{\circledR} 3800$, no Laboratório Nacional de Nanotecnologia (LNNano - CNPEM) com taxas de deformação de $10^{-4} \mathrm{~s}^{-1}, 10^{-2} \mathrm{~s}^{-1}$ e $1 \mathrm{~s}^{-1}$ e temperatura de $600{ }^{\circ} \mathrm{C}$. A simulação física que mais se assemelha a condição de impacto do receptáculo em alta temperatura $\left(600^{\circ} \mathrm{C}\right)$ é a de $1 \mathrm{~s}^{-1}$. Nesta condição foram encontrados os valores mais altos de limite de escoamento (368 MPa), resistência mecânica (482 $\mathrm{MPa}$ ) e alongamento (20\%). Com esta simulação, sugere-se que estes valores sejam utilizados no projeto mecânico do receptáculo para transporte de material radioativo.
\end{abstract}

\begin{abstract}
The duplex stainless steel UNS S32304 has been considered a candidate for the construction of a receptacle for the transport of radioactive material to meet future needs of the Brazilian Multipurpose Reactor (RMB) under construction. The receptacles must have high mechanical resistance to avoid failure and consequent exposure of the radioactive material, as considering the most critical condition an air accident with explosion. In this work the effect of different strain rates on the stress-strain behavior of UNS S32304 sheets with $1.8 \mathrm{~mm}$ thickness was evaluated, simulating an air accident. The samples were submitted to hot tensile tests in the Gleeble ${ }^{\circledR} 3800$ thermal-mechanical simulator at the Brazilian Nanotechnology National Laboratory (LNNano - CNPEM) with strain rates of $10^{-4} \mathrm{~s}^{-1}, 10^{-2} \mathrm{~s}^{-1}$ and $1 \mathrm{~s}^{-1}$ and temperature of $600^{\circ} \mathrm{C}$. The physical simulation that most closely the impact condition of the receptacle at high temperature $\left(600{ }^{\circ} \mathrm{C}\right)$ is that of $1 \mathrm{~s}^{-1}$. In this condition, were found the highest values of yield stress (368 MPa), ultimate tensile stress (482 MPa) and ductility (20\%). With this simulation, it is suggested that these values be used in the mechanical design of the receptacle for transporting radioactive material.
\end{abstract}

Keywords (Palavras chaves): Aço inoxidável duplex, Simulador termomecânico, Taxa de deformação, Deformação a quente.

\section{Introdução}

A maioria dos aços inoxidáveis duplex (AIDs) apresenta uma microestrutura bifásica composta por ilhas de austenita em uma matriz ferrítica em proporções aproximadamente iguais, 0 que thes confere a excelente combinação de propriedades mecânicas e de resistência à corrosão, tornando este material bastante atrativo do ponto de vista econômico [1-7].

Contudo, a exposição de um AID a temperaturas elevadas pode levar ao desequilíbrio termodinâmico 
com sequente precipitação de novas fases, levando ao desbalanceamento microestrutural [8].

Essas precipitações, que ocorrem numa faixa de temperatura de 300 a $1000^{\circ} \mathrm{C}$, tornam mais propensas a transformação da fase ferrítica em compostos intermetálicos (sigma, chi), nitretos, carbonetos e a fase $\alpha^{\prime}$, que podem levar a redução de tenacidade, ductilidade e da resistência à corrosão [1,3,9].

O transporte de materiais radioativos deve cumprir requisitos de segurança e proteção radiológica [10]. Visando atender as normas de segurança para utilização do AID UNS S32304 no desenvolvimento do receptáculo para transporte de materiais radioativos, foi estudado o efeito de diferentes taxas de deformação em alta temperatura, considerando como situação mais crítica um acidente aéreo com explosão.

Os corpos de prova foram submetidos a ensaios em um simulador termomecânico, que é capaz de reproduzir condições reais em um laboratório, através da combinação de ciclos mecânicos e térmicos, permitindo simular condições de processamento de materiais.

\section{Materiais e Métodos}

Utilizou-se tiras de um AID de baixa liga, caracterizado com UNS S32304 com espessura de 1,8 mm e 72,0 $\mathrm{mm}$ de largura. As tiras foram produzidas pelo processo de laminação a quente seguido de tratamento térmico a $1050{ }^{\circ} \mathrm{C}$ e resfriamento ao ar. A composição química foi caracterizada por fluorescência de raios $X$ (FRX) em um espectroscópio da marca Shimadzu, modelo EDX-720, e está exposta na Tabela 1.

Tabela 1: Composição química do aço inoxidável duplex UNS S32304 caracterizada por FRX

\begin{tabular}{cccccc}
\hline$\%$ Mn & $\% \mathrm{Cr}$ & $\% \mathrm{Ni}$ & $\% \mathrm{Mo}$ & $\% \mathrm{Cu}$ & $\% \mathrm{Fe}$ \\
\hline $1,57(2)$ & $22,55(1)$ & $3,47(1)$ & $0,24(2)$ & $0,36(1)$ & $73(1)$ \\
\hline
\end{tabular}

Com objetivo de confeccionar os corpos de prova, de acordo com o croqui fornecido pelo LNNano para atender as especificações da Gleeble ${ }^{\circledR}$ (Figura 1), peças de UNS S32304 foram desbastados lateralmente para atingir a dimensão de $90 \times 14 \mathrm{~mm}$ em um centro de usinagem da marca Romi, modelo D600, sendo que os furos foram realizados em furadeira de coluna manual da marca Kone, modelo KM32, e o raio de 20 $\mathrm{mm}$ foi usinado em uma fresadora ferramenteira da marca WMW. O corpo se prova já usinado encontra-se na Figura 2.

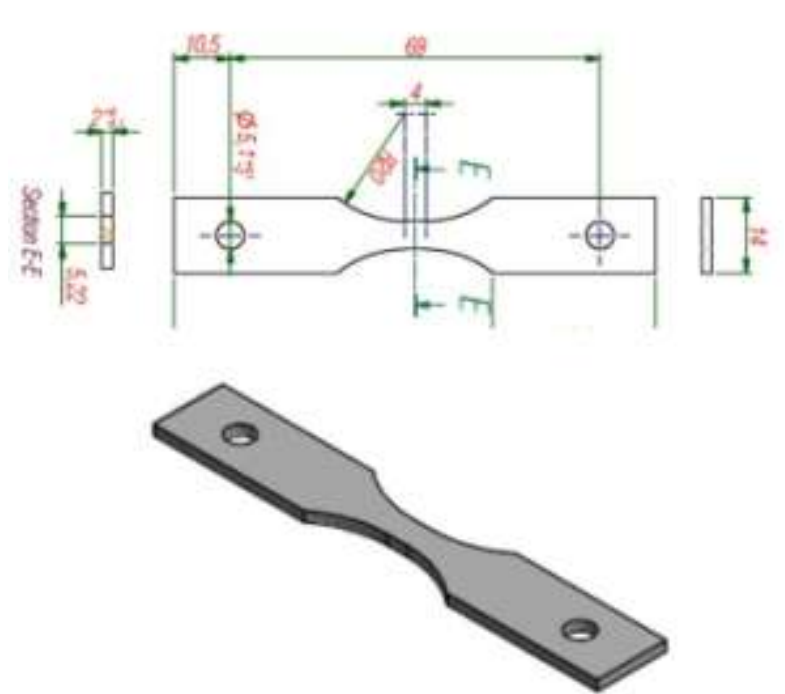

Figura 1: Dimensões do corpo de prova utilizado pelo LNNano na Gleeble $^{\circledR}$.

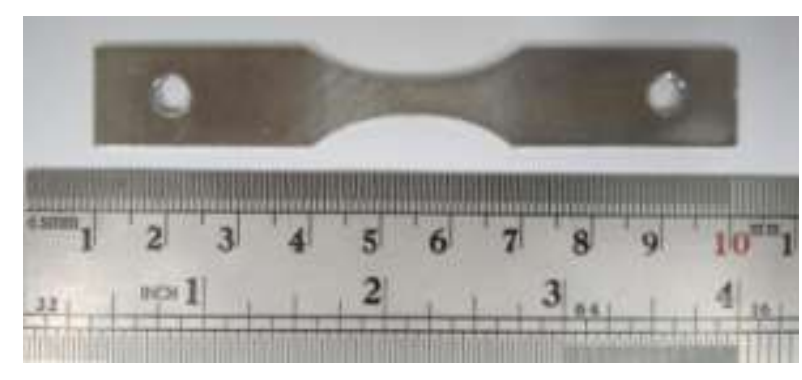

Figura 2: Corpo de prova usinado.

Ensaios de tração a quente foram realizados em um simulador termomecânico Gleeble ${ }^{\circledR} 3800$ instalado no Laboratório Nacional de Nanotecnologia (LNNano), localizado no Centro Nacional de Pesquisa em Energia e Materiais (CNPEM). O corpo de prova foi montado dentro de uma câmara bombeada a vácuo, com pressão mínima de $10^{-2}$ Torr. O simulador possui sistema hidráulico com capacidade de carga de $40 \mathrm{kN}$ em tração com resolução de $0,1 \mathrm{kN}$. O aquecimento é por efeito Joule, e fornece taxa de aquecimento de até $1000{ }^{\circ} \mathrm{C} / \mathrm{s}$. A temperatura foi medida por termopar tipo K. As amostras foram aquecidas até $600^{\circ} \mathrm{C}$ e tracionadas ao longo da direção de laminação até a fratura, com taxas de deformação de $10^{-4} \mathrm{~s}^{-1}, 10^{-2} \mathrm{~s}^{-1} \mathrm{e}$ $1 \mathrm{~s}^{-1}$, conforme esquema da Figura 3. 


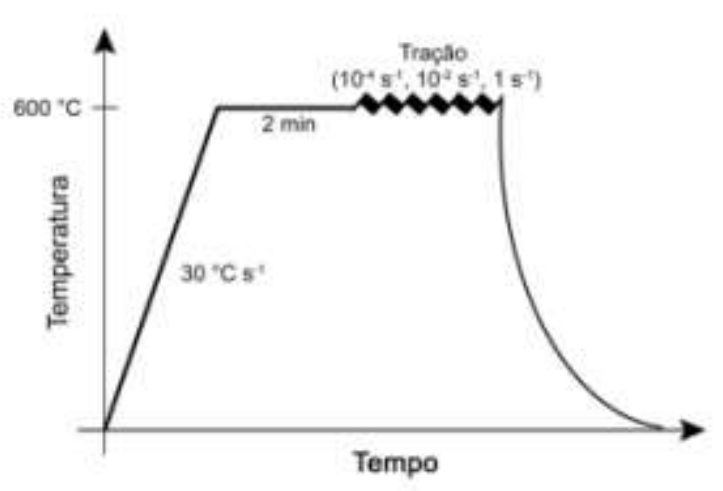

Figura 3: Esquema do ensaio realizado no simulador termomecânico Gleeble ${ }^{\circledR} 3800$, onde as amostras foram aquecidas até $600{ }^{\circ} \mathrm{C}$ e deformadas com taxas de $10^{-4} \mathrm{~s}^{-1}, 10^{-2} \mathrm{~s}^{-1}$ e $1 \mathrm{~s}^{-1}$.

\section{Resultados e Discussão}

A Figura 4 mostra as curvas Tensão-Deformação de engenharia obtidas nos ensaios de tração a quente para cada taxa de deformação estudada.

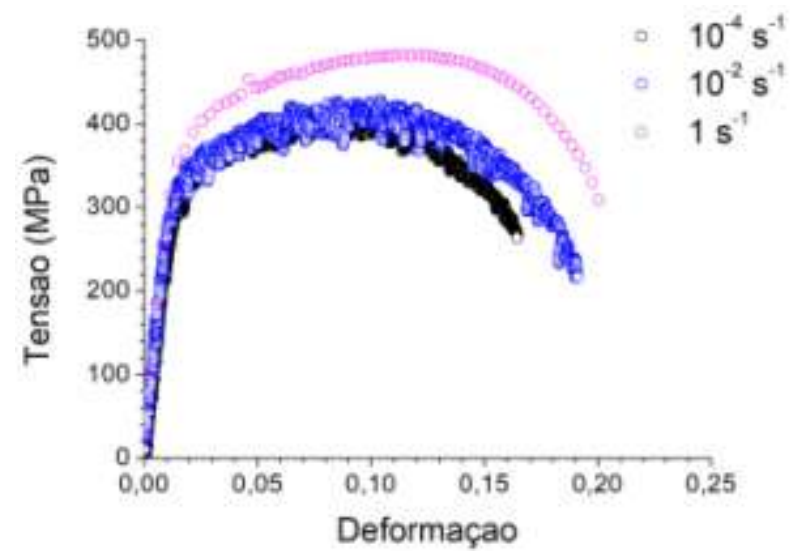

Figura 4: Curvas Tensão $x$ Deformação de engenharia das amostras deformadas a $600{ }^{\circ} \mathrm{C}$ com taxas de $10^{-4} \mathrm{~s}^{-1}, 10^{-2} \mathrm{~s}^{-1} \mathrm{e} 1 \mathrm{~s}^{-1}$.

As principais propriedades mecânicas obtidas a partir destas curvas são apresentadas na Tabela 2.

A Figura 5 mostra pontos experimentais com as respectivas curvas de tendência das principais propriedades mecânicas para as diferentes condições de deformação. Pode-se observar que as propriedades mecânicas melhoram à medida que a taxa de deformação é incrementada. Destaca-se que a maior taxa de deformação, $1 \mathrm{~s}^{-1}$, corresponde a simulação que mais se assemelha a condição de impacto em alta temperatura.
Tabela 2: Principais propriedades mecânicas das amostras deformadas a $600{ }^{\circ} \mathrm{C}$ com taxas de $10^{-4} \mathrm{~s}^{-1}, 10^{-2} \mathrm{~s}^{-1}$ e $1 \mathrm{~s}^{-1}$.

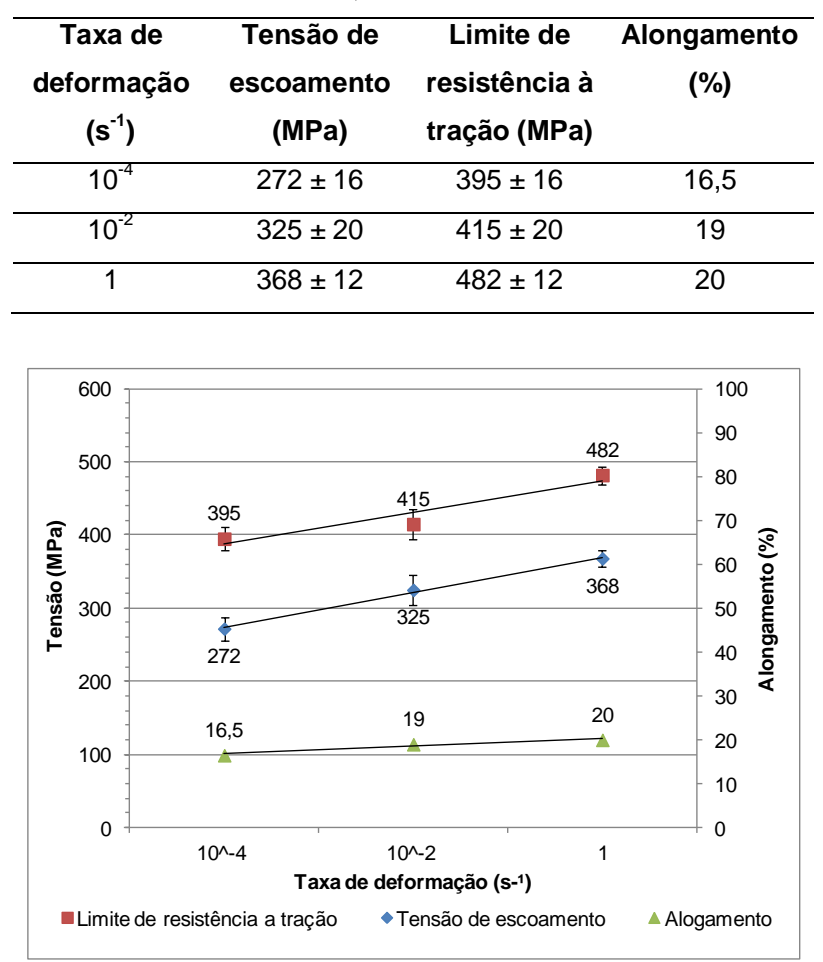

Figura 5: Curvas de tendência para as principais propriedades mecânicas das amostras deformadas a $600{ }^{\circ} \mathrm{C}$ com taxas de $10^{-4} \mathrm{~s}^{-1}$, $10^{-2} \mathrm{~s}^{-1}$ e $1 \mathrm{~s}^{-1}$.

\section{Conclusões}

A simulação física que mais se assemelha a condição de um acidente aéreo (impacto do receptáculo em alta temperatura, $600^{\circ} \mathrm{C}$ ) é a de maior taxa de deformação $\left(1 \mathrm{~s}^{-1}\right)$.

Nesta condição foram encontrados os valores mais altos de limite de escoamento (368 MPa), resistência mecânica (482 MPa) e alongamento (20\%).

Com esta simulação, sugere-se que estes valores sejam utilizados no projeto mecânico do receptáculo para transporte de material radioativo.

Este comportamento será investigado por difração de raios $X(D R X)$ com luz síncrotron (proposta ID: XTMS 20170659) no Laboratório Nacional de Luz Síncrotron (LNLS - CNPEM).

\section{Agradecimentos}

Os autores agradecem à CAPES - Pró-Estratégia 2224/2012 e ao CNPQ pelas bolsas e ao LNNano e 
CNPEM pelo apoio na utilização da Gleeble ${ }^{\circledR}$ (Proposta STC-22766).

\section{Referências}

[1] CHARLES, J. Duplex stainless steels, a review after DSS' 07 held in Grado. Revue de Métallurgie, Maastricht, p. 155-171, 2008.

[2] GUNN, R. N. Duplex stainless steel: microstructure, properties and application. Abington: Woodhead Publishing Ltd, 1997.

[3] IMOA. Orientações práticas para processamento dos aços inoxidáveis Duplex. International Molybdenum Association, Londres, 2012.

[4] SAHU, J. K. et al. Effect of $475 \circ \mathrm{C}$ embrittlement on the mechanical properties of duplex stainless steel. Materials Science and Engineering A, v. 508, p.1-14, 2009

[5] POHL, M.; STORZ, O.; GLOGOWSKI, T. Effect of intermetallic precipitations on the properties of duplex stainless steel. Materials Characterization, v. 58, p. 6571, 2007.
[6] BETINI, E. G.; MUSCI, C. S.; LUZ, T. S.; ORLANDO, M. T. D.; AVETTAND-FÈNOËL, M-N.; ROSSI, J. L. Effect of post-weld heat treatment on thermal diffusivity in UNS S32304 duplex stainless steel welds. Archives of Materials Science and Engineering, v. 88, p. 49-58, 2017.

[7] NILSSON, J.-O. Super duplex stainless steels. Materials Science and Technology, v.8, p. 685-700, 1992.

[8] CALLIARI, I.; BRUNELLI, K. ZANELLATO, M.; RAMOUS, E.; BERTELLI, R. Microstructural modification during isothermal ageing of a low nickel duplex stainless steel, J. Mater. Sci, v.44, p. 37643769, 2009.

[9] LONDOÑO, A. J. R. Precipitação de fases intermetálicas e austenita secundária na ZAC de soldagens multipasse de aços inoxidáveis duplex. Tese de doutorado. Escola Politécnica da Universidade de São Paulo, São Paulo, 2001.

[10] CNEN - COMISSÃO NACIONAL DE ENERGIA NUCLEAR. CNEN NE 5.01: Transporte de Materiais Radioativos - Resolução CNEN 013/88. Ministério da Ciência, Tecnologia e Inovação. Agosto, 1988. 